\title{
Improving the Believability of Non-Player Characters in Simulations
}

\author{
${ }^{1}$ Jere D. Miles and ${ }^{2}$ Rahman Tashakkori \\ Division of Business and Public Service Technologies, Wilkes Community College, Wilkesboro, NC 28697, USA \\ ${ }^{2}$ Appalachian State University \\ Department of Computer Science, Appalachian State University, Boone, NC 28608, USA \\ jere.miles@wilkescc.edu and ${ }^{2} \mathrm{rt} @$ cs.appstate.edu
}

\begin{abstract}
In recent years the video game industry has experienced rapid expansion developing virtual environments that accurately mimic a real-world setting. However, the industry almost entirely relies on finite state machines for deploying computer-controlled characters within these environments. This has resulted in simulated inhabitants that lack the same degree of believability as their surroundings. As part of this research a simulation was developed using Java in which an agent was placed. In a survey students were asked to rank the believability of different artificial intelligence techniques employed by the simulation. The genetic algorithm developed for this simulation provided for an agent whose decisions were more believable than the decisions generated by a finite state machine or random selection process.
\end{abstract}

\section{Introduction}

The notion of believability is explored within this paper through the implementation of a virtual environment that simulates a popular consumer video game inhabited by a computer-controlled character. The research is particularly interested in the role that different decision-making mechanisms may play in the observed believability of the character.

A hypothesis that incorporating evolutionary computing techniques, such as a genetic algorithm, within the agent's decision-making process may provide an increased believability of the agent over those utilizing more traditional video game artificial intelligence approaches was established. In order to test this hypothesis, a simulator was developed with three separate decisionmaking mechanisms: a random selector, a finite state machine, and a genetic algorithm. These mechanisms were observed in operation by human viewers who rated each on their believability as a human-like character within the virtual environment.

This research is significant as there is little or no publication exploring the potential differences between these artificial intelligence techniques in terms of perceived believability. Many researchers have documented the improvement that evolutionary techniques

Copyright (C) 2008, The Second Conference on Artificial General Intelligence (agi-09.org). All rights reserved. provide in a competitive or problem solving environment. Also, There have been documented efforts that have discovered some of the required characteristics to improve the believability of a computer-controlled character [3][5]. However, these efforts have not focused on the decisionmaking mechanism employed by the agent. This paper will address the potential usability of evolutionary artificial intelligence techniques within a video game environment. While exploring the usefulness of these methods, it was expected to demonstrate that these approaches were not only achievable, but that they would provide an increase in believability of the computer-controlled character to a human observer. This increased believability may provide for a more engaging interactive product that can be delivered by the video game industry

\section{Background}

The artificial intelligence systems of video games have historically relied on a finite state machine to provide a decision-making mechanism for the computer-controlled characters. These finite state machines are implemented in virtually all computer games that have been developed and released [1]. Recent additions have allowed scripting techniques to be used as a method to fine-tune the state machines that actually generate the logic for the agents [7]. It has been considered necessary for the video game to employ these techniques in order to ensure a stable and testable product.

However, in recent years, human players have begun a migration towards games that involve playing with other people rather than playing with a computer. This trend seems to be a result of human players finding the computer-controlled characters to be too predictable in their behaviors to offer a reasonable level of challenge. Also, other human players were preferred for their ability to be flexible and adapt their responses to dynamic situations [6]. There has been a renewed interest in the development of believable video game agents with the success of Electronic Arts' The Sims [2]. This series of games is considered to have raised the expectation of artificial intelligence within a video game and many developers are attempting to replicate a similar degree of believability [7]. The premise of The Sims is that the 
player plays the role of a virtual human making the decisions of this character's life [4]. A character that is not directly under the control of a human player generates its actions based upon the behaviors that are present in all of the objects [8].

\section{Results}

In order to test the impact that genetic algorithms may have on the believability of a computer-controlled character, a virtual environment was created to mimic many of the capabilities and displays of Electronic Arts' The Sims [2]. The simulator was named StudentLife and was populated by a computer-controlled student character, which was then observed in operation by human volunteers. The virtual environment did not allow these observers to interact with the system in any way other than through observance. This insured that the observers would focus on the behaviors of the agent being surveyed. The system allowed the human observers to have a full view of the virtual environment and all of the internal conditions of the agent.

Ninety-seven surveys were administered to the observers. In Figure 1, a comparison is provided of the highestranking values from each of the observers. Of the human observers, $43 \%$ found that the genetic algorithm behaved the most believably of the options. The remainder was split with the finite state machine preferred by $27 \%$ and the random selector rated the highest by $12 \%$. The remaining $18 \%$ of the observers gave a similarly high value to more than one of the logic engines on their survey.

\section{AI Logic Engine Preference}

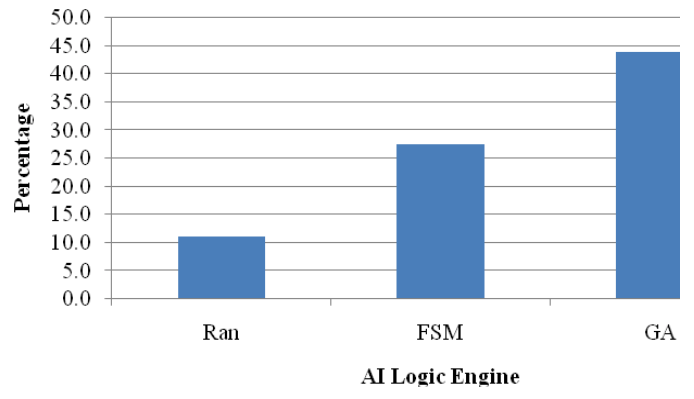

Figure 1: Logic engine preferences overall

It is worth noting that among the respondents, those who gave the random engine a higher score did so due to the variations in the activities that were performed. The observers expressed an interest in the believability of the random student because of the different actions that were performed, not because of the condition in which the agent was. This implied that for $11 \%$ of the respondents, the state of the agent's needs and happiness had no bearing on the believability of the behavior of the computer-controlled character.

\section{Conclusions}

From the results that were collected, it has been concluded that a genetic algorithm could be developed with the ability to govern the decision-making process for a computercontrolled character within a simulated environment. The concerns of a dumb intelligence evolving [7] were adequately addressed through the strength of the fitness function in combination with the mutation capability to introduce new genes to an evolving set of solutions.

Based upon the results of the surveys, it was found that a genetic algorithm provided a more believable virtual inhabitant. These results could be applicable to all video games that utilize agents for the purposes of story advancement or cooperative game play. Games that have an inherit need for a competition from the computer, may not gain an improvement in believability through these techniques.

\section{References}

[1] Buckland, Matt, 2005, Programming Game AI by Example, Woodware Gamed Developer's Library.

[2] Electronic Arts, 2008, http://thesims.ea.com.

[3] Laird, John, E., Duchi, John, 2000, "Creating HumanLike Synthetic Characters with Multiple Skill Levels," AAAI 2000 Fall Symposium Series: Simulating Human Agents.

[4] Macedonia, Michael, 2000, "Using Technology and Innovation to Simulate Daily Life," Computer, V. 33, No 4.

[5] Paiva, Ana, Dias, Joao, Sobral, Daniel, Aylett, Ruth, Sobreperez, Polly, Woods, Sarah, Zoll, Carsten, Hall, Lynne, 2004, "Caring for Agents and Agents that Care: Building Empathic Relations with Synthetic Agents," Proceedings of the Third International Joint Conference on Autonomous Agents and Multiagent Systems.

[6] Swetser, Penelope, Johnson, Daniel, Swetser, Jane, Wiles, Janet, 2003, "Creating Engaging Artificial Character for Games," Proceedings of the Second International Conference on Entertainment Computing.

[7] Woodcock, Steven, 2001, "AI Roundtable Moderator's Report," 2001 Game Developer's Conference.

[8] Wright, Will, Forbus, Kenneth, 2001, "Some Notes on Programming Objects in The Sims," Qualitative Reasoning Group. 